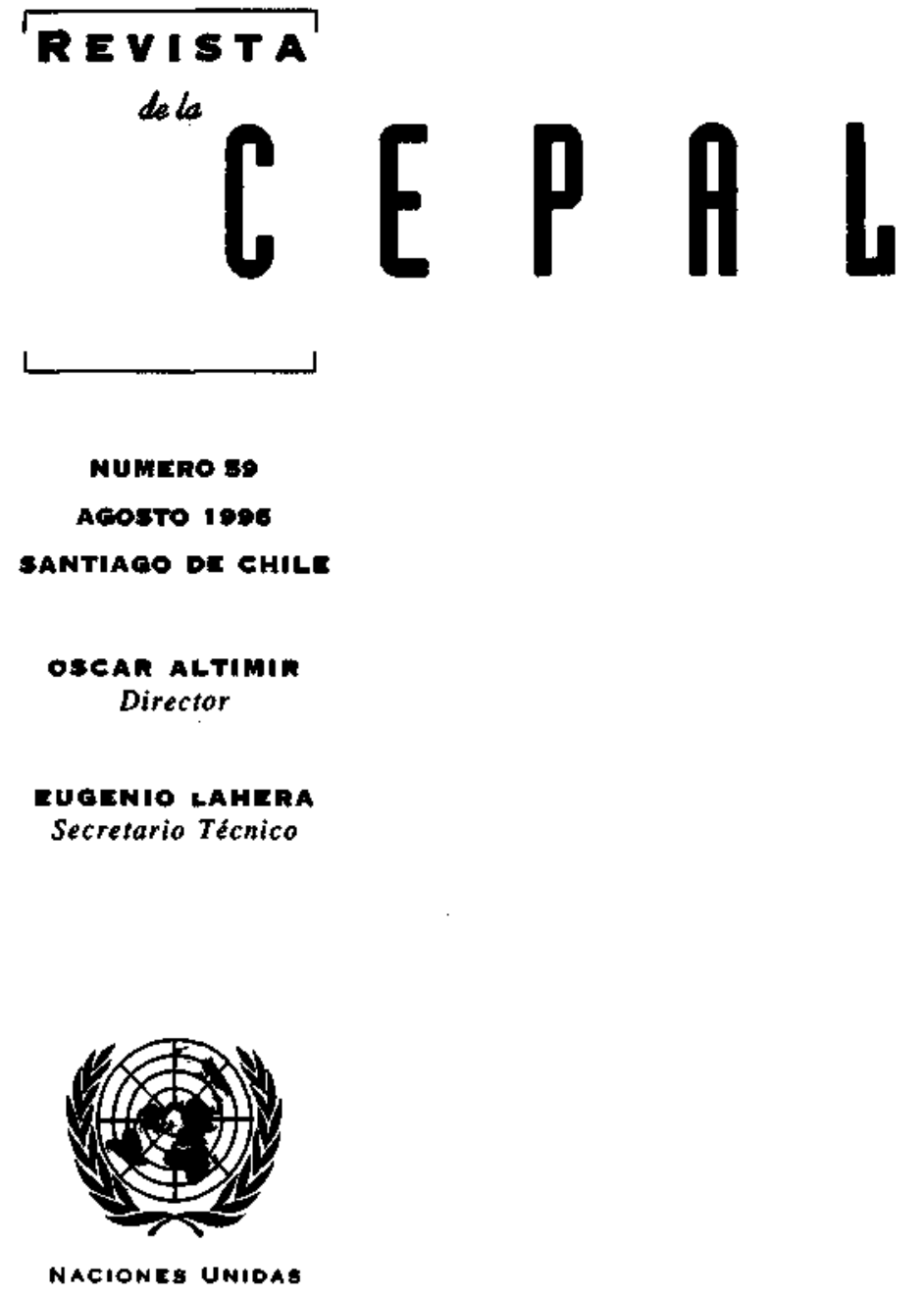


Interacciones entre macro y microeconomía

Gert Rosenthat

Politica económica, instituciones y desarrollo productivo en América Latina

Osvaldo Rosales

Competitividad sistémica: nuevo desafío para las empresas y la política

Klaus Esser, Wolfgang Hillebrand, Dirk Messner y Jörg Meyer-Stamer

La formación de habilidades báslcas y la capacitación para el trabajo productivo

Guillermo Labarca

Los fondos de inversión social en América Latina

Gabriel Siri

Mercados de derechos de agua: componentes institucionales

Miguel Solanes

Los países del Caribe y el área de libre comercio de las Amérlcas

Trevor Harker, Sidya Ould El-Hadj y Lucio Vinhas De Souza

La experiencia argentina: ¿desarrollo o sucesión de burbujas?

Hugo Nochteff

Posibilidades y límites de la reforma en la gestión de

la salud en Chile

Ana Sojo

Orientaciones para los colaboradores de la Revista de la CEPAL 


\section{Los países del Caribe y el área de libre comercio de las Américas}

\section{Trevor Harker* \\ Sidya Ould El-Hadj** Lucio Vinhas de Souza**}

* Asesor Económico Regional para el Caribe

** Oficiales de Asuntos

Económicos. Sede Subregional de la CEPAL en Puerto España
Los países del Caribe están muy conscientes de sus limitaciones de tamaño, ya sea que se les considere en función de uno o de todos los criterios de superficie, población o producto interno bruto (PIB). Esto, parad6jicamente, también los obliga a sumarse a un grupo comercial más numeroso por temor a que, de lo contrario, no se les permita participar plenamente en la actividad internacional. En este artículo se examinan varias características propias de los países de pequeño tamaño, sobre todo las que parecen especialmente pertinentes para el Caribe. Se observa que, de hecho, las limitaciones de tamaño plantean mayores exigencias a las autoridades nacionales en lo que respecta a la idoneidad y la coherencia de la gestión económica; además, hacen que los ciudadanos de los países pequeños vivan expuestos a un mayor nivel de riesgos, derivados de los caprichos del clima o bien de las turbulencias de los mercados internacionales. Se admite asimismo que la gama de opciones productivas que se abre a los países pequeños es de por sí ljmitada y que los costos de la transición necesaria para ingresar al área de libre comercio de las Américas (ALCA) bien pueden ser elevados, sobre todo porque algunos países del Caribe han aplicado políticas que de alguna manera son contrarias a las exigencias de esta nueva situación. Se hace hincapié en la necesidad de que muchos países pequeños se preparen mejor para acceder al ALCA y se formulan varias propuestas de política interna y externa que podrían adoptarse con ese fin. También se sugiere que, a fin de mitigar los costos de corto plazo de la transición y elevar la propia capacidad de participar en el ALCA, Jos países pequeños traten de asegurar en las negociaciones previas algunos arreglos de carácter transitorio centrados en las políticas internas, las medidas para aumentar la producción de bienes exportables y las estrategias de negociación conjunta. 


\section{I}

\section{Introducción}

En la Cumbre de las Américas celebrada en Miami en diciembre de 1994, los participantes se comprometieron a establecer un área de libre comercio de las Américas (ALCA) a más tardar en el año 2005, en la que se eliminarían progresivamente las barreras al comercio y la inversión en el hemisferio occidental.' Los participantes acordaron celebrar acuerdos sobre distintos temas, tales como barreras arancelarias y no arancelarias que afecten al comercio de bienes y servicios, agricultura, subsidios, inversion, derechos de propiedad intelectual, compras del gobierno, barreras técnicas al comercio, salvaguardias, reglas de origen, derechos antidumping y compensatorios, normas y procedimientos sanitarios y fitosanitarios, solución de controversias y política de competencia.

El carácter aperturista de esa área de libre comercio se puso de manifiesto en la promesa de los posibles países miembros de no levantar barreras al comercio y la inversión de otras naciones y reafirmar su compromiso con las reglas y disciplinas comerciales internacionales del Acuerdo General sobre Aranceles Aduaneros y Comercio y la Organización Mundial de Comercio (GATT/OMC). Se indicó que el objeto del ALCA era consolidar los extraordinarios logros que ya habían alcanzado los países del hemisferio en cuanto a la liberalización del comercio y la integración económica, que se consideran factores claves para el desarrollo sostenible.

En la Cumbre de las Américas también se señaló que las políticas ambientales y de liberalización comercial se apoyan mutuamente y que se aseguraría la observancia y promoción de los derechos de los trabajadores, tal y como están definidos en los convenios internacionales pertinentes. Sin embargo, se acordó evitar las restricciones encubiertas al comercio, de conformidad con los acuerdos del GATT/OMc. Con esta referencia se advirtió que el ALCA aún debía llegar a un entendimiento respecto del medio ambiente y de los derechos de los trabajadores en lo que afectan a la liberalización del comercio en el hemisferio, ya que estos temas seguramente volverían a surgir una vez concluidos los acuerdos especiales.

\footnotetext{
I Plan de Acción aprobado en la Cumbre de las Américas, realizada
} en Miani, Florida, del 9 al 11 de diciembre de 1994.
En la Reunión Ministerial sobre Comercio celebrada en Denver el 30 de junio de 1995 se aprobó un programa de trabajo para preparar el inicio de las negociaciones que culminarían en el ALCA que, según se expreso, representaría un compromiso global que integraría todas las obligaciones y derechos recíprocos. Se crearon varios grupos de trabajo para iniciar una labor de estudio de diversos temas especializados, uno de los cuales se encargará de formular propuestas para que las economías más pequeñas participen eficazmente en el ALCA.

Los preparativos con miras al ALCA se producen en el contexto de un entorno comercial mundial y hemisférico notablemente más libre. Los factores que contribuyen a esta situación son la implementación unilateral de políticas de liberalización comercial por parte de muchos países, la reciente popularidad de los acuerdos bilaterales y plurilaterales de libre comercio en la región y la inauguración del acuerdo GATT/OMC, que busca aclarar y fortalecer las normas y disciplinas que rigen la inversión y el comercio internacionales.

El ALCA abarca un gran número de posibles países miembros, ${ }^{2}$ que presentan grandes diferencias en cuanto a población, superficie, estructura económica, dotación de recursos, nivel de ingreso, niveles de conocimientos y de desarrollo tecnologico, calidad de la infraestructura básica y productividad. Estas diferencias son acusadas entre los países industrializados y algunos de los países en desarrollo que podrían ser miembros de la agrupación propuesta. Sin embargo, también se advierten diferencias significativas entre los propios países en desarrollo, que incluyen economías grandes y pequeñas con distintos grados de desarrollo.

El hecho de que un país sea pequeño no constituye en sí una desventaja económica, aunque por cierto limita la gama de opciones de desarrollo a que pueden recurrir las autoridades, dado que los países pe-

\footnotetext{
${ }^{2}$ Los siguientes 34 países participaron en la Cumbre: Antigua y Barbuda, Argentina, Bahamas, Barbados, Belice, Bolivia, Brasil, Canadá, Chile, Colombia, Costa Rica, Dominica, Ecuador, El Salvador, Estados Unidos de América, Granada, Guatemala, Guyana, Haití, Honduras, Jamajca. México, Nicaragua, Panamá, Paraguay, Perí, República Dominicana, Saint Kitts y Nevis, Santa Lucía, San Vicente $\mathrm{y}$ las Granadinas, Suriname, Trinidad y Tabago, Unguay y Venezuela.
} 
queños tienen economías intrínsecamente abiertas que dependen mucho del comercio exterior. Esto significa que las políticas que fomentan una interacción eficiente con la economía internacional se ven ampliamente recompensadas, mientras que las otras son severamente castigadas. Tanto a escala mundial como en el Caribe es posible encontrar ejemplos de ambos tipos. Entre los primeros, cabe destacar Singapur, la provincia china de Taiwán, Hong Kong o Luxemburgo, mientras que en la región del Caribe también hay economías pequeñas, algunas muy pequeñas, que han alcanzado niveles de vida relativamente altos gracias al comercio de servicios turísticos y financieros: las Bahamas y las Islas Caimán son dos buenos exponentes.

Empero, son pocos los países del Caribe que sistemáticamente han seguido el camino de la apertura económica. Los que han intentado aplicar políticas de sustitución de importaciones al amparo de elevados aranceles no han registrado niveles semejantes de crecimiento económico, dado que la expansión se ha visto limitada por la escasez crónica de divisas. Los ingresos de exportación han sido exiguos, vulnerables a los vaivenes de los mercados de exportación y, en algunos casos, las industrias han llegado a depender del trato preferencial para poder sobrevivir. Ahora estos países deberán hacer frente a un riguroso proceso de ajuste para revertir decenios de políticas inadecuadas, a fin de mitigar el impacto adverso que tendrá en sus economías la apertura de los mercados y aprovechar las oportunidades que ésta les brindará en el largo plazo.

Los países de la Comunidad del Caribe (CARICOM) están haciendo esfuerzos por reformar las políticas comerciales regionales y las macroeconómicas internas. No obstante, el ritmo de la reforma de la política macroeconómica interna ha sido desigual en estos países, ya que en algunos casos las políticas regionales han cambiado más lentamente que las políticas internas y han dejado de ser coherentes con ellas. Las tensiones también se han hecho evidentes a medida que algunos países han intentado acelerar la reforma de las políticas comerciales regionales, lo que se ha manifestado por ejemplo en las diferentes posturas con respecto al ritmo y grado de reducción del arancel externo común y al alcance de la ampliación del mercado, y se ha reflejado en diferencias de opinión acerca de la Asociación de Estados del Caribe y el AlcA.

A un nivel más general, las economías pequeñas presentan ciertas características que, sin ser exclusivas de ellas, pueden plantearles problemas especiales al tratar de alcanzar el desarrollo económico y social. Por ejemplo, registran altos costos unitarios en materia de infraestructura y administración pública, y la fragilidad de sus economías repercute negativamente en su gestión macroeconómica.

Las economías pequeñas tienen una base económica relativamente poco diversificada y dependen mucho de las exportaciones de unos cuantos productos y servicios básicos (cuadro 1). Por ejemplo, en 1994 las exportaciones de banano representaron $49.3 \%$ de las exportaciones de mercancías de Santa Lucía y $47.9 \%$ de las de Dominica. En el caso de Jamaica y Trinidad y Tabago se advierte una dependencia aún mayor de la bauxita y el petróleo, respectivamente. Por consiguiente, estos países pequeños son especialmente vulnerables a las fluctuaciones de los precios y la demanda de sus exportaciones. También registran altos niveles de importación, tanto para el consumo como para la producción, con lo que quedan a merced de las variaciones de la demanda y los precios mundiales. En todos los casos, el comercio de bienes sobrepasa el $50 \%$ del PIB. Si se incluyen los servicios no atribuibles a factores, la proporción que representa el comercio exterior es notablemente mayor, y en 1994 oscilo de casi el $153 \%$ del producto en Antigua y Barbuda a un $62 \%$ en Trinidad y Tabago. Las exportaciones, especialmente en la agricultura, tienden a concentrarse en los mercados en que los productos del Caribe reciben trato preferencial. Dados los altos niveles de protección que soportan algunas de las exportaciones de estos países, esa dependencia del comercio exterior ilustra su excesiva vulnerabilidad ante perturbaciones externas, como los cambios de política.

Las reducidas dimensiones de los mercados internos impiden que las empresas que operan en ellos aprovechen las economías de escala o los crecientes rendimientos a escala en aquellas industrias sensibles a dicho efecto (De Vries, 1984). Inversamente, cuando intentan aprovechar dichas economías, se ven obligadas a dedicarse desde un principio a los mercados de exportación, lo cual las priva de la posibilidad de contar con un mercado interno conocido en el cual perfeccionar su capacidad. En realidad las industrias que se desarrollaron en el Caribe tienden a estar sumamente protegidas y su producción se destina sobre todo al mercado interno, $o$ al mercado regional en el caso de los países miembros de la CARICOM. ${ }^{3}$ Todo intento de reducir los aranceles se complica porque algunos países dependen en gran medida de los impuestos sobre

\footnotetext{
${ }^{3}$ En Centroamérica no existen los mismos niveles de protección (véase Lewis, 1994).
} 
CUADRO I

Países del Caribe: Algunos indicadores para 1994

(Porcentajes)

\begin{tabular}{|c|c|c|c|c|c|}
\hline Países & $\begin{array}{c}\text { Concentración } \\
\text { de exportaciones }\end{array}$ & $\begin{array}{c}\text { Comercio de } \\
\text { mercancías' } \\
\text { PlB }\end{array}$ & $\begin{array}{c}\text { Mercancías y } \\
\text { servicios no } \\
\text { atribuibles a } \\
\text { factores/PIB }\end{array}$ & $\begin{array}{l}\text { Impuestos al } \\
\text { comercio/ } \\
\text { recaudación } \\
\text { fiscal }\end{array}$ & $\begin{array}{c}\text { Tasas de } \\
\text { desempleo }\end{array}$ \\
\hline Anguila & .. & 55.4 & 146.9 & 77.0 & \\
\hline Antigua y Barbuda & & 75.8 & 152.6 & 66.1 & 5.7 \\
\hline Barbados & 23.3 & 54.8 & $95.7^{b}$ & 8.6 & $2 !, 2^{\mathrm{c}}$ \\
\hline Belice & 34.1 & 79.2 & 128.0 & 54.4 & 9.8 \\
\hline Dominica & 47.9 & 79.3 & 124.5 & 56.5 & 9.9 \\
\hline Granada & 9.3 & 58.6 & 99.1 & 56.9 & 15.3 \\
\hline Jamaica & $78.4^{\text {đ }}$ & 78.6 & $101.9^{\mathrm{h}}$ & 14.0 & $15.4^{\mathrm{c}}$ \\
\hline Montsertat & $\ldots$ & 41.5 & $77.3^{b}$ & 52.5 & 5.5 \\
\hline \multicolumn{6}{|l|}{ República } \\
\hline Domtnicana & .25 .3 & 32.9 & 62.7 & 44.8 & \\
\hline Saint Kitts y Nevis & 40.7 & 65.2 & 144.5 & 55.2 & 4.7 \\
\hline Santa Lucía & 49.3 & 69.3 & 113.9 & 58.7 & 7.2 \\
\hline San Vicente y las Granadinas & 29.0 & 70.0 & 90.5 & 50.6 & 17.9 \\
\hline Suriname & 57.4 & 77.1 & 92.8 & 41.7 & \\
\hline Trinidad y Tabago & $75.5^{t}$ & 55.8 & 61.9 & 10.4 & $18.5^{\mathrm{c}}$ \\
\hline
\end{tabular}

Fuente: CEPAl, sobre la base de información oficial.

a Principales exportaciones, como porcentaje de las exportaciones de mercancías.

b Corresponde a ingresos procedentes de bienes y turismo solamente.

c Corresponde a 1994; el resto se basa en los datos más recientes.

d Corresponde a bauxita y alúmina.

e Corresponde a 1993.

f Corresponde a petróleo y petroquímica.

el comercio exterior para la obtención de ingresos. Los derechos de importación representan más de la mitad del ingreso tributario de los países de la Organización de Estados del Caribe Oriental (OECo) y Belice, y en Antigua y Barbuda constituyen hasta un $66 \%$. Barbados, Jamaica y Trinidad y Tabago, que han aplicado políticas de reforma tributaria, dependen mucho menos de los impuestos sobre el comercio exterior y en 1994 registraron porcentajes del 9 al 14\%, aproximadamente. En Granada, empero, las primeras medidas encaminadas a reformar el régimen impositivo quedaron contrarrestadas por las dificultades para recaudar los impuestos al consumo.

Las islas del Caribe también son propensas a sufrir desastres naturales, como los huracanes. Por su reducido tamaño y la consiguiente especialización en unos pocos grupos de productos, un solo huracán basta para eliminar gran parte de los ingresos de exportación. Además, el frágil ecosistema de los pequeños Estados insulares los hace más vulnerables que otros a la degradación ambiental.

La apertura recíproca de los mercados propuesta en el marco del aLCA y sus posibles consecuencias entrañan algunos problemas especiales para las economías pequeñas, entre ellos sobre todo la probabilidad de que se elimine el trato preferencial, y el consiguiente aumento de la competencia a que se verán sometidos en el hemisferio productos que antes gozaban de proteccion. En alguna medida las economías pequeñas consideran injusta su situación en los mercados internacionales, porque se ven obligadas a competir con países más desarrollados que poseen una notable superioridad en materia de conocimientos, tecnología y productividad. Por lo tanto, desde esta perspectiva, dicha competencia conducirá inexorablemente a un desplazamiento de sus exportaciones y a la extinción de sus industrias de sustitución de las importaciones. Irónicamente, los países industrializados también se están pronunciando contra la apertura de los mercados a los países menos desarrollados, porque temen que los bajos salarios y las normas laborales y ambientales imperantes en los países en desarrollo -que ellos juzgan indulgentes- provoquen el cierre de empresas, la baja de los salarios y el aumento del desempleo en el mundo desarrollado.

Tanto en la Cumbre de las Américas, de Miami como en la Reunión Ministerial sobre Comercio, de Denver se reconocieron las grandes diferencias de tamaño que existen entre los posibles miembros del ALCA, y los países se comprometieron a buscar los medios 
para proporcionar oportunidades tendientes a facilitar la integración de las economías más pequeñas y aumentar su nivel de desarrollo. Así pues, los participantes estaban conscientes de los complejos problemas que suscita la división de costos y beneficios en el área de libre comercio propuesta, en particular en lo que se refiere a las economías pequeñas.

El presente artículo tiene por objeto aclarar algunas de estas cuestiones. En él se analizan varias de las medidas adoptadas por distintas agrupaciones comerciales y de integración para corregir el problema de la desigualdad de los beneficios para los países miembros. También se analizan los posibles costos y beneficios que tendría la participación en el ALCA para las pequeñas economías. Por último, se proponen algunas medidas de política que podrían ayudar a los países más pequeños a ajustarse a la expansión del mercado hemisférico.

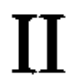

\section{Modos de abordar la desigual distribución de los beneficios en las distintas agrupaciones comerciales y de integración}

Generalmente está aceptado en la literatura y refrendado por la experiencia que los beneficios de una agrupación comercial no siempre son parejos. En consecuencia, en los distintos acuerdos comerciales regionales se ha prestado considerable atención a la forma de compensar a quienes tienen menos posibilidades de acceso a los beneficios. En general, si bien los acuerdos de financiamiento, como los fondos especiales, parecen ofrecer beneficios tangibles y duraderos, no está tan claro que sea así en el caso de los diferentes requisitos de ingreso. Sin embargo, los criterios utilizados por la Unión Europea (UE), la CARICOM y el Tratado de Libre Comercio de Norteamérica (TLCN) son útiles para orientar a los países pequeños en su preparación para las negociaciones con miras al ALCA. Cabe señalar, no obstante, que la UE es un mercado único que está en proceso de convertirse en una unión política, mientras que la CARICOM tiene elementos tanto de unión aduanera como de mercado común y el TLCN es una zona de libre comercio. Los distintos objetivos propios de estos niveles de integración tal vez conduzcan a diferencias en los enfoques con que ellos abordan la desigualdad en la distribución de los beneficios, aunque todos constituyen buenos ejemplos de las distintas opciones posibles.

\section{El trato que dispensa la Unión Europea a los países menos desarrollados}

Básicamente se usan dos mecanismos (concesiones), para abordar el problema de la diversidad estructural dentro de la UE. ${ }^{4}$ La primera concesión es un período de transición para permitir que el país miembro adapte la legislación y las normas nacionales a las prácticas y niveles de la ue; esto significa que los sectores considerados particularmente débiles en determinados países pueden tardar varios años en competir en pie de igualdad con los de otros países miembros de la Unión. ${ }^{5}$ La segunda concesión, paralela y a menudo de mayor duración que la primera, guarda relación con los llamados fondos estructurales de la uE. Pese a que el objetivo de reducir las diferencias entre las regiones de la Comunidad se menciona en el preámbulo mismo del Tratado de Roma de 1957, dichos fondos se crearon en el marco de las declaraciones del Acta Unica Europea sobre la "solidaridad de mercado", de 1988. Su creación también se vio impulsada por el aumento de la diversidad estructural de la ue tras su segunda ampliación, en que se incluyo a Grecia, en 1981, y la tercera, en que se incluyó a España y Portugal, en $1986 .^{6}$ En sus distintas formas, los fondos representan un

\footnotetext{
${ }^{4}$ Los paises seleccionados para recibir trato especial son España, Grecia, Irlanda y Portugal.

5 Sectores concretos como la banca y las finanzas se beneficiaron de "periodos de transición" de hasta siete años en España y Portugal. La política agrícola común (PAC) también es una concesión, aunque se aplica a toda la Unión y tiene carácter "permanente". - La importancia de los fondos estructurales puede apreciarse simplemente por su tamaño: 60000 millones de ECU, o to que es lo mismo, 80000 millones de d6lares para el quinquenio 1989-1993. La magnitud de los fondos también puede medirse en relación con las economias de los países receptores: en el periodo 1989-1993 representaron el $2.3 \%$ del PIB de Grecia, el $2.9 \%$ del de ltlanda y el $3.5 \%$ del de Portugal.
} 
esfuerzo real de cumplir con los criterios de convergencia mediante mecanismos que aumentan la eficiencia económica con el fin de propiciar condiciones de mercado allí donde no existen y facilitar la provisión de bienes públicos escasos.

\section{Trato dispensado a los paises miembros me- nos desarrollados en la caricom}

El principio del trato especial para los países miembros menos desarrollados ${ }^{7}$ también está sólidamente establecido en la CARICOM, en la que se cré́ un régimen especial a fin de otorgarles un trato preferencial en el proceso de integración. Se les concedió un período de ajuste de 10 años para aplicar el arancel externo común, el doble del plazo que se les dio a los países miembros más desarrollados. También disfrutaron de un perfodo de gracia de 10 años para participar en el libre comercio con estos últimos, y se les permitió además aplazar la adopción del arancel externo común para fomentar sectores nacionales determinados, y suspender el trato de mercado común para las importaciones de los países más desarrollados cuando la aplicación de dicho trato redundara en grandes pérdidas de ingresos. Además, los países menos desarrollados recibieron concesiones especiales en cuanto a las normas de origen, en virtud de las cuales, a los grupos de productos más destacados en su estructura de producción, como la fruta procesada y las chapas galvanizadas, se les otorgó trato de mercado común en condiciones favorables. En este caso el porcentaje de insumos extranjeros se fijó en un nivel superior al de los países más desarrollados (Banco Mundial, 1990). A los países menos desarrollados también se les permitió otorgar subvenciones directas e indirectas a la exportación en el comercio regional para favorecer a los productores nacionales. Por último, el sistema de incentivos fiscales armonizados para la inversión permitió que los países menos desarrollados otorgaran a los inversionistas exenciones arancelarias y períodos de gracia más largos, habitualmente de 15 años, mientras que en el caso de los países más desarrollados éstas eran de sólo 10 años.

\section{El trato dispensado a México en el TLCN}

Pese a los principios de reciprocidad con arreglo a los cuales se negoció el TLCN, México consiguió que se le otorgara trato diferenciado en algunas áreas. Por ejemplo, se acordaron períodos distintos de escalonamiento, para proteger a México de súbitos incrementos de las importaciones provenientes de sus socios más desarrollados. El acuerdo estipulaba que la proporción de importaciones mexicanas que ingresarían libres de impuestos equivaldría como máximo al $43 \%$ de sus importaciones no petroleras desde los Estados Unidos y al $41 \%$ del mismo tipo de importaciones desde Canada. ${ }^{8}$ Sin embargo, el $84 \%$ del total de exportaciones no petroleras de México a los Estados Unidos y el 79\% de las mismas exportaciones a Canadá tendrían acceso a estos países, libres de impuestos, a partir de enero de 1994.

En el caso de los textiles, se acordó que los Estados Unidos comenzaría por eliminar los aranceles en el $45 \%$ de sus importaciones desde México, mientras que México sólo tendría que eliminar aranceles en el $20 \%$ de sus importaciones de textiles desde los Estados Unidos. También se negociaron concesiones con México para el sector automovilístico, en virtud de las cuales aproximadamente el $81 \%$ del valor de las exportaciones mexicanas de repuestos a los Estados Unidos ingresarían libres de impuestos una vez que el TLCN entrara en vigor, mientras que sólo $5 \%$ de las exportaciones estadounidenses de partes y componentes ingresarían a México libres de impuestos. En cuanto a la agricultura, se convino en que los Estados Unidos eliminaría los aranceles aplicados a productos agrícolas importados desde México que correspondan al $61 \%$ del valor de las importaciones estadounidenses de ese rubro. Por su parte, México eliminaría los aranceles aplicados a productos agrícolas importados desde los Estados Unidos que correspondan al $31 \%$ del valor de las importaciones de ese rubro (Sera Puche, 1992). No obstante, se pretendía que estas concesiones fueran temporales y en el acuerdo se estipuló un plazo limitado durante el cual se produciría la liberalización del comercio en todos los sectores.

\footnotetext{
${ }^{7}$ Dentro de la caricom los que reciben trato de país menos desarrollado son Antigua y Barbuda, Belice, Dominica, Granada, Saint Kitts y Nevis, Santa Lucía y San Vicente y las Granadinas. Los países más desarrollados son Barbados, Guyana, Jamaica y Trinidad y Tabago.
}

\footnotetext{
${ }^{8}$ Estos porcentajes se basan en el valor del comercio de 1991.
} 
III

\section{Posibles beneficios de la apertura}

\section{de los mercados}

Es bien sabido, aunque no esté universalmente aceptado, que el libre comercio aumenta la eficiencia y el bienestar. Para decirlo sencillamente, el comercio permite que un país se especialice en las actividades que puede realizar de manera más productiva, maximizando sus utilidades al intercambiar una parte de su producción. A su vez, las importaciones liberan al país de la obligación de producir todos los bienes y servicios que necesita para sobrevivir, muchos de los cuales sería físicamente incapaz de producir, mientras que gran parte del resto sólo podría producirlos a un costo muy elevado. Gracias al libre comercio, la producción del país puede concentrarse en las actividades más eficientes, en tanto que las demás necesidades nacionales pueden cubrirse recurriendo a quienes realizan dichas actividades en forma más competitiva en el exterior. Así pues, el bienestar se maximiza con el comercio, dado que todas las partes se benefician. Si bien esta teoría se originó en los países desarrollados, se adecua más a los países pequeños y menos desarrollados, porque son ellos los que pueden resultar más favorecidos con el comercio. Sin embargo, los países pequeños tienen una necesidad relativamente mayor de contar con un acceso seguro al mercado y reglas comerciales estables y transparentes para poder desempeñarse con eficacia.

En reconocimiento de estos principios se formó el GATT, para promover el desarrollo del comercio multilateral y definir y aplicar las reglas que deberían regirlo. Actualmente ha asumido este papel la organización sucesora del GATT, la omc. Desde la creación del GATT surgieron varias agrupaciones comerciales regionales. Si bien se apartaban de los principios multilaterales puros, generalmente el GATr las aceptaba, considerándolas como una alternativa admisible. ${ }^{9}$ Esta aceptación obedecía en parte al hecho de que los acuerdos comerciales regionales podían impulsar a los países

\footnotetext{
${ }^{9}$ En el Artículo 24 del Acuerdo se explican las condiciones en que se considera que la integración no infringe lo dispuesto en las reglamentaciones del GaTT. Posteriormente se hicieron esfuerzos, que culminaron en la OMC, para definir con mayor precisión dichas condiciones.
}

miembros hacia la meta del multilateralismo con más rapidez que lo que se podría haber logrado unilateralmente. El ALCA también encierra este potencial, dado que podría establecer una zona de libre comercio que abarcaría a la mayor parte del comercio de bienes y servicios en casi todos los países del hemisferio. La otra opción - lograr unilateralmente el mismo grado de liberalización del comercio a través del GATT/OMCtiene menos posibilidades de materializarse, habida cuenta del clima actual. Esta perspectiva es especialmente válida en el caso de los países más pequeños, ya que algunos de ellos se agrupan en torno a acuerdos comerciales limitados con los que se hallan firmemente comprometidos desde hace mucho tiempo. Además, no todos los países están dispuestos a aceptar los principios del comercio multilateral ni los riesgos consiguientes.

Actualmente la liberalización del comercio está reapareciendo gracias a la acción de algunos de los países del Caribe que experimentaron el desequilibrio económico más grave en los años ochenta. Para ellos, y para todas las economías pequeñas, los ingresos de exportación desempeñan un papel primordial en su expansión económica, por lo que su incremento se convirtió en fundamental para cumplir con las obligaciones que imponía la deuda externa. El éxito de otros países pequeños, que han basado su crecimiento en las exportaciones, ${ }^{10}$ contribuyó a reforzar este principio. Para establecer el equilibrio y lograr un crecimiento sostenido se decidió mejorar el desempeño del sector de exportaciones. Se aplicaron políticas para favorecer la producción de bienes y servicios transables, comenzando con medidas destinadas a aumentar la eficiencia económica y reducir los distintos sesgos contrarios a las actividades exportadoras. Estas medidas se iniciaron con la aplicación de políticas monetarias y fiscales restrictivas y reajustes del tipo de cam-

\footnotetext{
${ }^{10}$ Sobre todo en Asia, donde la liberalización del comercio se aplicó de manera indiscriminada, en lugar de hacerlo a través de mecanismos de comercio regionales. De esta manera se evitaron los peores errores de la industrialización mediante la sustitución de importaciones, que en el mejor de los casos sólo tuvo una utilidad muy limitada dentro de zonas de mercado de pequeffas dimensiones.
} 
bio real. Posteriormente, se modificaron con frecuencia los regímenes cambiarios, para que el mercado desempeñara un papel más importante en la determinación del tipo de cambio, esperando así evitar los graves desajustes de las monedas que se habían producido cuando éste se fijaba administrativamente. Una vez que se contuvo la demanda interna, los gobiernos de esos países se sintieron en situación de concentrarse en varias políticas de mediano plazo, como los códigos de inversión extranjera y la liberalización del sistema financiero. El tema de las políticas del mercado laboral aún no se ha abordado de manera sistemática; sin embargo, ello parece justificarse en vista de las altas tasas de desempleo crónicas de algunos países. No obstante, el diálogo entre la parte patronal y los sindicatos ha contribuido a una mejor comunicación entre los interlocutores sociales.

También se prestó atención al régimen comercial, haciéndose hincapié en la eliminación de los sesgos contrarios a la exportación que aún persistían en la estructura arancelaria y en los procedimientos administrativos que regían el comercio. Pero, a escala nacional, el ritmo de la reforma fue desigual en algunos casos, ya que las políticas de integración existentes, basadas en el consenso, habitualmente dificultaban los avances en aquellos temas sobre los que había una postura regional común. En varios casos la política comercial iba a la zaga de otras políticas macroeconómicas internas y era fuente de tensiones. A menudo se pasaban por alto las iniciativas o se demoraba su ejecución, lo que creaba frustración en la agrupación regional entre los paŕses que deseaban seguir adelante con la reforma económica.

Es probable que el ingreso en grupo al sistema más amplio del ALCA constituya un medio más viable de reducir las barreras al comercio entre los países pequeños. ${ }^{11}$ Este enfoque tiene la ventaja de brindar cierta continuidad con las políticas anteriores y permite mantener la solidaridad entre los miembros actuales y conservar parte de la seguridad que supuestamente otorgaban los acuerdos vigentes. También se considera como una manera de dar un mayor margen de ma-

\footnotetext{
1 Muchos consideran que la creación de la Asociación de Estados del Caribe es una etapa intermedia en la formación del mercado hemisférico más amplio, originalmente a través del $\mathrm{TLCN}$, y más recientenrente a través del ALCA. Aunque debido a problemas de calendario podría complicarse la situación, la Asociación aún podría ser el instrumento para que los países unificaran sus políticas, aumentaran su poder de negociación y se habituaran a la competencia en un grupo más numeroso de países del Caribe, antes de su plena incorporación al acuerdo hemisférico más amplio.
}

niobra en materia de política económica a quienes buscan maximizar la eficiencia económica e imprimir el máximo impulso a las exportaciones. Al mismo tiempo, la ampliación aumenta el tamaño del mercado, lo que algunos estiman necesario para mantener el ímpetu de las exportaciones.

El acceso a un mayor mercado se considera ventajoso porque el de la Caricom es de por sí pequeño y se ve aún más limitado por las políticas de gestión de la demanda necesarias para mantener el equilibrio frente a la atonía del sector de exportaciones. El mayor tamaño del mercado, junto con la aplicación de aranceles uniformemente bajos y normas de origen flexibles, reducirá la incidencia de la desviación del comercio y permitirá aprovechar las economías de escala. El acceso a un mercado ampliado, en el marco de un acuerdo formal como el ALCA, seguramente mitigará la incertidumbre que encierra la reglamentación del comercio de bienes y servicios y proporcionará mecanismos transparentes para la solución de controversias. Todos estos factores son enormemente beneficiosos para los países pequeños, que no tienen poder de mercado que los sostenga en caso de disputas comerciales. ${ }^{12}$ Por último, para un país pequeño expuesto a cambios de tendencia en su política interna, el hecho de encuadrar las variables fundamentales en el marco de un acuerdo multilateral puede tener la ventaja de aumentar la coherencia de la política. Por consiguiente, la liberalización del comercio, que ha encontrado resistencia en algunos países del Caribe, se podría fortalecer o acelerar gracias a la participación en el ALCA.

Es probable que la recompensa que se obtendría de las mayores economías de escala, la mayor seguridad y transparencia de los mercados y la mayor coherencia de la política, sumada a las medidas de liberalización del mercado que ya se han iniciado unilateralmente, se traduzca en un aumento de la inversión interna y externa y de la transferencia de tecnología para las empresas del sector exportador. Al mismo tiempo, es improbable que un acuerdo comercial como el aLCA -dados sus bajas tasas arancelarias y el gran tamaño de su mercado- obstaculice la penetración en el mercado mundial de los pequeños países que tengan tal posibilidad. A plazo mediano y largo, el ALCA podría elevar los niveles de crecimiento económico, empleo y equidad.

\footnotetext{
12 Por ejemplo, Estados Unidos utiliza la Ley general de comercio y competitividad de 1988 para decidir unilateralmente y aplicar, 0 amenazar con aplicar, sanciones comerciales contra los países cuya conducta comercial considera desleal.
} 
La iniciativa de consolidar el mercado único dentro de la CARICOM podría sufrir algunas presiones derivadas de los esfuerzos tendientes al ingreso al ALCA, ya que los gobiernos del Caribe han decidido llevar adelante ambos procesos simultáneamente. Una justificación del mercado único de la CARICOM es el argumento de la industria incipiente, es decir, que el mercado protegido estimulará la inversión en empresas más grandes y eficientes para la producción de bienes y servicios, lo que a la larga hará que la región esté en mejores condiciones de penetrar en mercados más amplios como la Asociación de Estados del Caribe y el ALCA. A este argumento se contrapone el hecho de que el ingreso al ALCA exigirá una notable reducción de los aranceles vigentes en la CARICOM que, para ser manejable, debería escalonarse y comenzar pronto. Así pues, se observa una evidente falta de coherencia entre el argumento de la industria incipiente y la necesidad de que la transición al mercado más amplio se produzca sin sobresaltos. Desde un punto de vista más práctico, actualmente el ritmo relativo de convergencia hacia el mercado único es tal que quizá éste no entre en vigor con la suficiente rapidez para que esta expectativa se materialice. Por lo tanto, es probable que en el mercado único, con aranceles que siguen siendo altos, los participantes tengan dificultades para alcanzar a tiempo un nivel de competencia en la producción que les permita aprovechar las ventajas de la apertura del mercado. Por último, el argumento a favor de la protección tiende a hacer caso omiso de su corolario: los sesgos contrarios a la exportación que ponen en desventaja a las nuevas industrias que deben ingresar a los mercados competitivos. Por estas razones, no habría que esperar a la creación del mercado único para reducir rápidamente el arancel externo común.
El segundo argumento es que un mercado único y consolidado de factores de producción ofrece horizontes más vastos a la hora de producir. Se ha llegado a un consenso amplio para reducir los obstáculos que dificultan el flujo de capital y de ciertas categorías de mano de obra calificada en el mercado único, pero la legislación nacional para poner en práctica estas decisiones se ha aprobado con lentitud. Ambas actividades seguramente traerán consigo beneficios a largo plazo para la región y no quedarán contrarrestadas ni limitadas por el aumento esperado de la inversión extrarregional que se obtendrá con la ampliación del mercado. Es probable que este beneficio se deje sentir aunque los aranceles sigan siendo altos, de modo que no es en absoluto incompatible con la ampliación del mercado.

El tercer argumento en favor de profundizar la integración de la CARICOM es que se lograría una mayor coordinación de las políticas externas. Una posibilidad sería la negociación conjunta para ingresar al ALCA como grupo. En cierto sentido, ello podría ser ventajoso para la CARICOM, dado que preservaría mejor la integridad del grupo ${ }^{13}$ y permitiría que se resolvieran conjuntamente los problemas de interés común. También simplificaría las negociaciones, para todas las partes, si todos los países miembros de la CARICOM participaran como una sola entidad. Frente a estos beneficios hay que ponderar el riesgo de que el ritmo desigual de la reforma económica dentro de la CARICOM complique los preparativos que se están llevando a cabo en cada país con miras a las negociaciones, y comprometa las posibilidades de ingreso de todo el grupo, a menos que se logren rápidos progresos en los temas que puedan resultar más conflictivos, por ejemplo, el arancel externo común.

\section{IV}

\section{Posibles costos de la apertura de los mercados}

Los temores expresados por las economías más pequeñas y menos desarrolladas respecto de su integración en zonas económicas más amplias se pueden clasificar en dos grandes categorías: los de índole económica y los de índole política. En el plano político, las naciones temen la pérdida de soberanía que implica cualquier compromiso internacional que tenga repercusiones internas. Las naciones más pequeñas, que a me- nudo acaban de independizarse, son las que tienen mayor recelo a este respecto, dado que están profundamente conscientes de su escaso poder de negociación. Sin embargo, a medida que proliferan las transaccio-

\footnotetext{
13 Para continuar con toda la gama de actividades beneficiosas en esferas distintas del comercio que quedarán pendientes después del ingreso al ALCA.
} 
nes internacionales, es prácticamente inevitable que todos los países experimenten cierta pérdida de soberanía. Una manera de responder al problema de la limitación del poder de negociación podría ser que las naciones más pequeñas negocien como un bloque regional, aunque esta estrategia también supone cierta pérdida de soberanía. Por otra parte, su pequeño tamano podría ayudarles a obtener concesiones que las naciones más grandes no se atreverían a solicitar.

La cuestión política de la pérdida de soberanía también puede tener repercusiones económicas, aun en compromisos aparentemente sencillos. Por ejemplo, en un informe sobre el TLCN se indica que todo país que desee suscribir un acuerdo de libre comercio con Estados Unidos debe comprometerse a mantener un entorno macroeconómico estable ${ }^{14}$ Esta exigencia conlleva un límite en la aplicación de las políticas anticíclicas que prefieren algunos gobiernos, aun cuando no se establezcan indicadores explícitos y estrictos como los estipulados en los criterios de convergencia de la UE. Asimismo, los acuerdos complementarios del TLCN, especialmente los que se refieren a las prácticas de trabajo y las normas ambientales, pueden aminorar el atractivo que ofrecen algunos países para la inversión y entrañar costos significativos para su aplicación en los países pequeños.

En el aspecto económico, a estos países les preocupan, sobre todo, las pérdidas que puede provocar la apertura de la industria nacional a la competencia extranjera y los costos de adaptación al mercado ampliado. Esta preocupación se advierte especialmente en los países del Caribe y Centroamérica que actualmente operan con acuerdos de mercado común. La literatura económica tradicional se ocupa más de los beneficios derivados de la apertura del mercado que de las pérdidas. Esos beneficios tienen su origen en una asignación más eficiente de los recursos, que genera una producción y un nivel de bienestar más altos. Aun así, los costos de la transición son reales y es preciso tenerlos presentes.

En general, se considera que las posibles pérdidas se limitan al corto plazo, dada la existencia de algunos factores de producción que son específicos: por ejemplo, trabajadores capacitados para desempeñar tareas especializadas o fábricas construidas para producir ciertos bienes. Los factores de producción específicos son los que están vinculados a un proceso de producción determinado, que puede dejar de ser com-

14 Véase Report on the North American Free Trade Agreement, volúmenes I y II (G.S.R. Associates, Puerto España, 1994). petitivo con la apertura de la economfa, y cuya aplicación a otros usos no es fácil ni inmediata. Sin embargo, se supone que en el largo plazo todos los factores de producción acaban por ajustarse a las señales del mercado, es decir, son susceptibles de usos alternativos adecuados, que permiten llegar finalmente a una situación de equilibrio optimo. En este argumento se reconoce que siempre habrá sectores relativamente competitivos, ya que en el marco de la teoría ricardiana de la ventaja comparativa, si bien es posible que un país sea menos competitivo en términos absolutos en todos los sectores, es imposible para cualquier país ser relativamente menos competitivo en todos ellos. Así pues, aunque en términos absolutos todos los sectores sean menos competitivos que los mejores del mundo, todos los agentes nacionales pueden, en potencia, salir beneficiados con el comercio, dado que los sectores que en términos relativos son más competitivos pueden generar los recursos necesarios para compensar las posibles pérdidas de los sectores relativamente menos competitivos a través de distintos mecanismos de transferencia. ${ }^{15}$

Al analizar los efectos comerciales de la integración económica, los economistas generalmente consideran tres categorías: creación de comercio, desviación del comercio y efectos globales del comercio. La creación de comercio consiste en establecer nuevas corrientes comerciales entre los países miembros de la nueva zona económica que desplazan a la costosa producción nacional anterior; la desviación del comercio se define como la sustitución de importaciones caras provenientes de los países miembros por importaciones baratas de terceros países. El efecto global del comercio es el resultado de los dos factores anteriores y representa el efecto comercial neto que produce la zona de comercio preferencial sobre sí misma y sobre el resto del mundo. ${ }^{16}$ Desde el punto de vista del bienestar mundial, todo tipo de integración que no sea de carácter mundial es subóptima.

Hasta el momento son pocos los estudios realizados sobre los efectos concretos del TLCN en las economías más pequeñas. Según uno de ellos, las nuevas condiciones de mercado que se han ofrecido a los

\footnotetext{
15 La clásica excepción a la regla es el famoso caso del "crecimiento pauperizante" (immiserizing growth), elaborado por Bhagwati en su trabajo de 1958. Hoy se considera en gran medida una posibilidad teórica. Véase Krugman y Obstfe]d, 1988.

16 Véase Markheim, 1994. En este estudio se calcula que el efecto comercial neto de la integración sobre las importaciones estadounidenses del Caribe entre 1987 y 1993 fue un aumento del $99.9 \%$, es decir, que el incremento fue creado prácticamente en su totalidad por las preferencias de la lniciativa para la Cuenca del Caribe.
} 
productores de textiles mexicanos no han repercutido negativamente en el sector textil centroamericano. En cambio sí se han producido efectos negativos derivados de la competencia entre los países centroamericanos en un sector orientado hacia las exportaciones con productos homogéneos y una alta elasticidad de sustitución. En este caso particular, el efecto neto del comercio fue nulo para las economías que estaban fuera del bloque comercial. ${ }^{17}$

En otro estudio, en el que se citan estimaciones de un informe del Banco Mundial, se pronostican pérdidas estáticas y dinámicas limitadas para los países de la CARICOM por no pertenecer al TLCN. Las pérdidas estáticas se concentraban en ciertos países y sectores, por ejemplo, Jamaica y los sectores de vestuario y calzado. Sin embargo, la mayor parte de los efectos del TLCN se advertían en las estimaciones dinámicas -es decir, las que incorporaban los efectos a largo plazo de un mercado mayor y la asignación más eficiente de los recursos-, al preverse que todos los países del Caribe excluidos de la zona de comercio unificada registrarían una clara reducción en sus tasas de crecimiento (Mc Intyre, 1994).

Como primera aproximación, podemos suponer que los sectores industriales menos competitivos que se desarrollaron en el marco de la estrategia de sustitución de las importaciones e imposición de altos aranceles serían los más afectados por la competencia externa en el corto a mediano plazo. Lo mismo podría decirse de los sectores agrícolas protegidos. Sin embargo, es probable que las estructuras arancelarias de protección se desmantelen gradualmente como consecuencia de las iniciativas del GATT/OMC.

Las conclusiones con respecto al sector de los servicios son algo menos claras. El sector turístico ya es relativamente abierto y competitivo, aunque algunos servicios auxiliares del sector podrían ser vulnerables a la competencia externa. ${ }^{18} \mathrm{La}$ apertura del sector financiero también podría significar el desplazamiento de algunas empresas nacionales, o al menos dar lugar a alguna forma de asociación con empresas ex-

\footnotetext{
${ }^{17}$ Esto significa que en el cotto plazo probablemente dichos países podrian seguir siendo competitivos en el sector, pero una vez que uno de ellos entra en el área de comercio preferencial en forma unilateral, todos los que quedan fuera pierden. La estrategia optima para ellos sería negociar e ingresar como bloque regional (Véase CEPAL, 1995).

18 No obstante, es probable que la plena integración de Cuba en el sector turístico de la region ocasione cambios enormes en la participación en el mercado que le comesponde a cada país.
}

tranjeras. ${ }^{19}$ Los servicios de telecomunicaciones en la mayoría de los países son de propiedad extranjera o se explotan en régimen de empresa conjunta con compañías extranjeras. Sin embargo, es posible que, con la incorporación de nuevos partícipes a este sector -que tiene una marcada tendencia al monopolio, especialmente en los países pequeños - así como al sector de servicios financieros, aumente la eficiencia, y el cambio tecnológico sea más rápido. Por último, cabe señalar que la Ronda Uruguay de las negociaciones del GATT abarcó parcialmente los servicios y, en consecuencia, es probable que la OMC intensifique la liberalización de este sector.

Es posible que también se planteen otras inquietudes con respecto a: i) la importancia fiscal de los impuestos a la importación en algunos países de la región, dado que todo proceso de armonización que modifique significativamente los aranceles podría ocasionar dificultades presupuestarias a corto plazo, o hacer necesaria la apiicación de las consiguientes medidas de reforma tributaria; ii) la incompatibilidad con las estructuras preferenciales existentes, siendo el ejemplo más citado, aunque ni mucho menos el único, la incompatibilidad de la Convención de Lomé o la Iniciativa para la Cuenca del Caribe con una zona hemisférica de libre comercio, si bien una vez más esta estructura ya está en tela de juicio debido a la forma en que evoluciona la Ue y a los compromisos del GATT/OMC ya mencionados; iii) los costos vinculados con la armonización de patentes y derechos de autor, que podrían suponer un aumento de las transferencias financieras al exterior y la pérdida de libertad para los agentes internos que deseen establecer nuevos negocios, aunque ésta también es una cuestión que se abordará a escala mundial, a través del GATT/OMC; y iv) el costo financiero y humano que conlleva la administración de toda organización de integración, aunque en el caso del ALCA se prevé que será relativamente moderado. ${ }^{20}$

\footnotetext{
${ }^{19}$ En general se considera que las economías de escala son menos importantes en el sector financiero, en el que la existencia de una amplia red de sucuisales y el conocimiento local de las condiciones del mercado interno actúan como una barrera para frenar la entrada de competidores externos de importancia. Sin embargo, las reducidas dimensiones de los mercados financieros internos indican que la inversión fija en la red es relativamente pequeña, mientras que el interes cada vez mayor en las operaciones en el extranjero podría reducir la importancia relativa del conocimiento del mercado interno.

${ }^{20}$ Por ejemplo, el presupuesto de la UE lo financian todos los Estados miembros con cargo a una porción de su impuesto sobre el valor agregado (IVA) y de sus derechos de importación, hasta un monto equivalente al $1.21 \%$ del PIB agregado de la Unión para 1993. La mayor parte de estos recursos se destina a la política agrícola común (PAC) y a los fondos estructurales.
} 
En suma, algunas de las preocupaciones que tienen las naciones más pequeñas y menos desarrolladas respecto de las pérdidas a corto plazo que ocasionaría un proceso de integración hemisférica son legítimas, pero es preciso señalar que la mayoría de estas pérdidas se producirían en cualquier caso, ya que obedecen a factores históricos de carácter político, económico y tecnológico que afectan a todos los países. Si bien lo más probable es que el ALCA acentúe estas tendencias, el verdadero problema con que se enfrentan los países más pequeños es cómo adoptar durante el período de transición programas que amortigüen las perturbaciones más intensas, y les permitan llevar a cabo las medidas necesarias para mejorar su eficacia dentro del grupo.

\section{V}

\section{Propuestas de política}

La Reunión Ministerial sobre Comercio celebrada en Denver, en su declaración final, reconoció los intereses especiales de los países pequeños y aceptó buscar "activamente los medios para proporcionar oportunidades tendientes a facilitar la integración de las economías más pequeñas y aumentar su nivel de desarrollo". Además, encomendó al grupo de trabajo correspondiente que identificara los factores que afectan a la participación de los países más pequeños en el proceso del ALCA. ${ }^{21}$ Se examinarían los medios para facilitar su ajuste a tal proceso, la ampliación de su comercio y la inversión, y medidas para aumentar su capacidad negociadora. También se deberían identificar todos los demás aspectos de interés especial para las economías pequeñas, a fin de que pudieran ser considerados en las negociaciones.

Actualmente muchos de los países pequeños se benefician de acuerdos de comercio preferenciales que impulsan sus economías pero al mismo tiempo inhiben el proceso de ajuste necesario para su eficaz incorporación a un acuerdo de libre comercio más amplio. Este fenómeno se observa sobre todo en las exportaciones agrícolas tradicionales, como bananos y azúcar, pero también en algunos segmentos de la industria del vestuario y la mayoría de las manufacturas ligeras y los productos agroindustriales que se destinan al mercado regional. En casi todos los países más pequeños el efecto acumulativo de estas preferencias se traduce en una grave distorsión de sus estructuras de producción y consumo. No obstante, se ha hecho lo posible por llevar adelante el proceso de ajuste, incluso bajando el arancel externo común, con lo cual se han reducido

\footnotetext{
21 Declaración conjunta final de la Reunión Ministerial sobre Comercio (Denver, Colorado, 30 de jutio de 1995).
}

algunas de estas distorsiones. Sin embargo, en los casos en que las preferencias individuales cobran gran importancia en la economía, el panorama macroeconómico subyacente puede estar lo bastante distorsionado como para que los países retrasen la adopción de medidas encaminadas a ajustar los tipos de cambio reales y estimular la inversión en bienes exportables no protegidos. Al mismo tiempo, los efímeros beneficios derivados de la protección pueden ser tan grandes para la economía y la sociedad que las medidas de política se concentren en tratar de preservarlos en lugar de proceder al ajuste.

Una manera de romper este círculo vicioso es considerar por separado los beneficios financieros derivados, por ejemplo, de la protección del banano, y los de su producción. Esto sería posible si las preferencias, que tienen una vida finita, pudieran eliminarse progresivamente, por ejemplo en un período de cínco años, y el monto correspondiente a la preferencia reducida cada año se transfiriera a un fondo de inversión. Este fondo podría utilizarse para aumentar la eficiencia de la industria protegida o para invertir en otros bienes exportables. Dicha reducción de la preferencia alentaría a los sectores menos productivos a empezar a modificar de inmediato su producción, mientras que el menor volumen de ingresos de exportación provenientes de la preferencia podría invertirse en otros bienes exportables. Aun así, es posible que este mecanismo siguiera creando algunas distorsiones en la economía, de manera que habría que actuar con prudencia para garantizar que se seguirá adelante con las medidas de ajuste, prestar más atención al tipo de cambio real y propiciar de cualquier otra manera un clima de inversión propicio. En los casos en que la protección proviniera de un acuerdo interno (por ejemplo, un mecanismo de mercado común), se podría prestar al- 
gún tipo de asistencia, incluso en materia de reforma tributaria si procede, que estaría condicionada a la reducción más rápida de las preferencias que se estimen excesivas.

En un plano más general, los niveles de protección podrían ser tan elevados y la capacidad de respuesta a los cambios tan débil en los países más pequeños, que para ajustarse éstos quizá necesiten un plazo más largo que los países más desarrollados o de mayor tamaño del hemisferio. Aun en esos casos, habrá que seleccionar beneficios que no obstaculicen el proceso de ajuste, sino que lo estimulen. En todos los casos deberá reducirse la protección, en forma escalonada pero bastante rápida, aunque con períodos prolongados para prestar asistencia suplementaria. Siempre es preferible que sea la asistencia y no las altas tasas preferenciales lo que se mantenga durante períodos prolongados, ya que la protección obstaculiza el ajuste y alienta un sesgo contrario a la exportación, mientras que los incentivos financieros pueden usarse para facilitar la transición. Además de los fondos de inversión, la asistencia podría tomar la forma de recursos destinados a mejorar la educación básica, la capacitación -especialmente de los jóvenes-, la capacidad de gestión y el espíritu empresarial, así como el readiestramiento y demás medios para ayudar a los trabajadores de los sectores desplazados a encontrar nuevos empleos.

Los países pequeños padecen de por sí limitaciones de oferta y, como se indico anteriormente, en la mayoría de los casos los incentivos a la producción ofrecidos en el pasado han hecho que el producto resulte caro e inadecuado. Así pues, la primera prioridad debe ser la inversión de nuevos recursos en la producción de bienes transables. Sin embargo, en los casos en que se ha logrado una producción exportable o potencialmente exportable se necesitará asistencia inicial en materia de comercialización para aprovechar las ventajas de una nueva zona de comercio ampliada. Esta asistencia podría materializarse de distintas maneras, desde la representación - por parte de empresas comerciales del sector privado- de una gama de productos de países pequeños en los principales mercados, hasta medidas destinadas a estimular la inversion, incluso en forma de iniciativas conjuntas con fines de producción y comercialización.

Los problemas ambientales y los derechos de los trabajadores también revisten especial importancia para los países pequeños. Sin embargo, no deben utilizarse para imponer cargas desproporcionadas a su producción o erigir barreras a sus exportaciones. Las barre- ras bajas al comercio y la eficiencia económica que seguramente brindará el ALCA ayudarán a muchos países pequeños a obtener los recursos y el acceso a la tecnología necesarios para mejorar el medio ambiente. Es probable también que estimulen la inversión y el empleo, promoviendo así los derechos de un gran porcentaje de la fuerza de trabajo que actualmente está desempleada, lo que contribuirá en gran medida a aumentar la equidad social. Pese a que la desregulación del mercado quizá no baste para alcanzar todas las metas relativas al medio ambiente y a los derechos de los trabajadores, es conveniente recurrir en la medida de lo posible a las instituciones multilaterales de más amplia cobertura encargadas especialmente de resolver estos asuntos.

Por último, una limitación importante que sufren los países pequeños es su escasa capacidad para llevar adelante eficazmente las negociaciones previas a la firma de un complejo tratado internacional. En ellos el sector público cuenta con pocos recursos y la fuerza de trabajo calificada es escasa, de manera que es posible que, al celebrar negociaciones minuciosas, sobre todo en grupos de trabajo simultáneos, queden sin defender algunos intereses o se dispersen demasiado los esfuerzos con la consiguiente pérdida de eficacia. Una solución de probada utilidad para este problema logístico es que los países pequeños se unan y formen equipos negociadores conjuntos y que, colectivamente, contraten consultores bajo los auspicios de alguna institución común. Otra solución puede ser alentar a los agentes privados a ayudar a definir $-\mathrm{y}$, si procede, a defender- sus intereses.

Así pues, son varias las iniciativas que pueden adoptarse para mejorar la participación de los países pequeños en el ALCA; entre otras las siguientes, en su conjunto o combinadas de distintas formas:

i) Se podría introducir -0 acelerar la aplicación- de políticas internas destinadas a aumentar la capacidad de reacción de las economías pequeñas, a fin de que éstas respondan mejor a los acontecimientos externos, y a contribuir a que las empresas nacionales sean más competitivas en los mercados extranjeros.

ii) Se podría armonizar o acelerar la armonización de políticas en el marco de los respectivos acuerdos de integración, para hacerlas más coherentes con los objetivos de política interna anteriormente mencionados.

iii) Dadas la vulnerabilidad y la relativa falta de preparación de algunos Estados pequeños para hacer frente a un fuerte aumento de la competencia, éstos 
podrían tratar de negociar su ingreso en el mercado ampliado en forma escalonada, para que los productores locales dispongan de un período más largo de adaptación y reconversión. Estas concesiones deberían ser finitas y de duración limitada para que los paises adopten con más urgencia las medidas pertinentes. También deberían tener un carácter gradual, de manera que pueda absorberse eficazmente su impacto y que las economías se fortalezcan progresivamente y puedan adaptarse a ellas.

iv) Habida cuenta de que algunos países pequeños aún dependen altamente del proteccionismo, se podría conseguir financiamiento, en el contexto de una eliminación gradual acelerada de las preferencias, para aumentar cuando sea posible la productividad de las exportaciones tradicionales o estimular la producción de otros bienes susceptibles de exportación.

v) Puesto que los países pequeños registran elevados costos unitarios en la provisión de infraestructura básica, incluidos los servicios públicos, se podría intentar conseguir recursos especiales para financiar actividades que aumenten la productividad de la economía en su conjunto (por ejemplo, modernizar la infraestructura economica y desarrollar la capacitación profesional y la educación básica). vi) También se podría tratar de obtener de los países con mercados más grandes que ayuden a los países pequeños a aumentar la inversión en bienes exportables, propiciar empresas conjuntas o acuerdos de distribución de la producción, y crear mecanismos para comercializar sus exportaciones.

vii) En vista de las limitadas posibilidades de producción de los países pequeños, debería permitírseles cierta flexibilidad en cuanto a las normas de origen para fomentar la producción y el comercio de bienes y servicios.

viii) Los Estados pequeños también podrían beneficiarse si formaran un grupo negociador conjunto para definir sus intereses comunes más importantes y concordar respecto a ellos, planificar sus estrategias, preparar con cuidado su posición negociadora y participar conjuntamente en las negociaciones para defender sus intereses comunes.

ix) El sector privado debería participar plenamente en los preparativos y las negociaciones con miras al ALCA, dado que el resultado de las medidas que se apliquen para ampliar la oferta de bienes exportables -condición esencial para participar efectivamente en mercados más amplios- dependerá de su decidido apoyo y de su pericia para alcanzar el éxito.

\section{VI}

\section{Resumen y conclusiones}

Los países pequeños del Caribe enfrentan un riguroso proceso de ajuste como preparación para ingresar al ALCA. La tarea se complica porque ellos no están seguros de su propia capacidad de competir en los mercados mundiales, principalmente por la situación de desventaja en que los coloca su tamaño; y se dificuita aún más porque algunos países tendrán que adoptar medidas de política que se contraponen a prácticas aceptadas en los últimos 30 años. Finalmente, también frena el proceso el que Ios costos del ajuste probablemente se sentirán de inmediato, mientras que los beneficios seguramente tardarán más.

Tradicionalmente las economías del Caribe han estado estrechamente integradas con la economía mundial, y es lógico que así sea, por su pequeña magnitud y porque se ven obligadas a recurrir a las compras en el exterior para cubrir la mayoría de sus necesidades nacionales. Las medidas de política que se han aplicado en el pasado para tratar de atenuar esta dependencia externa han resultado contraproducentes, ya que a tal punto han distorsionado las estructuras de producción y deteriorado el desempeño del sector de exportación, que en algunos casos la única forma de mantener el intercambio con otros países ha sido mediante acuerdos de comercio preferenciales. La creciente globalización a través del GATT/OMc hace pensar que los acuerdos preferenciales irán siendo eliminados. La aparición de fenómenos paralelos a escala regional, especialmente la formación de agrupaciones comerciales más numerosas, exige también que se eliminen dentro del grupo las barreras que obstaculizan el comercio de bienes y servicios, las corrientes financieras y la tecnología. En cualquier caso, las presiones para lograr la apertura de los mercados del Caribe serán 
intensas. Por ese motivo, y en previsión de tales tendencias, varios países han iniciado un proceso de liberalización de los mercados.

Los preparativos para ingresar al ALCA son compatibles con las medidas que los países del Caribe deberán adoptar para integrarse eficientemente en los mercados mundiales más amplios. En el proceso que culminará con el ingreso al ALCA se espera que los países pequeños del Caribe puedan negociar un conjunto sistemático de medidas, calendarios y salvaguardias que les ayuden a llevar a cabo el ajuste. El acceso a un mercado más amplio claramente definido contribuirá segurarnente a eliminar parte de la incertidumbre que sienten los posibles inversionistas con respecto a la política económica y los mercados, acelerando así el ritmo de la inversión necesaria para transformar estas economías. Este proceso brinda a los países pequeños la oportunidad de prepararse mejor en un entorno en el que el riesgo es algo menor.

Como los países más pequeños están peor preparados y, por lo tanto es más probable que tengan dificultades para aprovechar los posibles beneficios del ALCA, deberían hacer algunos arreglos de carácter transitorio que les ayuden a mitigar los efectos de corto plazo de su incorporación. Estos arreglos deberían centrarse en tres categorías de acciones: medidas de política interna, medidas para aumentar la producción de bienes exportables y estrategias de negociación conjunta. Por lo que respecta a la primera categoría, los países deberían intentar establecer calendarios más flexibles, de manera que las políticas nacionales y, si procede, las políticas del respectivo grupo comercial, puedan adaptarse a las exigencias del ALCA. También deberían negociar la obtención de financiamiento especial en sustitución de las actuales preferencias de exportación, con miras a eliminarlas por completo. Para aumentar la producción de bienes exportables, debería crearse un fondo de desarrollo que permita aumentar la eficiencia de la economía en su conjunto, especialmente en esferas como la infraestructura y la capacitación. Los países pequeños también deberían solicitar asistencia para emprender iniciativas conjuntas, establecer acuerdos de distribución de la producción y desarrollar técnicas de comercialización; además, dadas sus limitadas posibilidades de producción y la importancia de obtener a tal efecto los insumos más eficientes desde el punto de vista de los costos, sería deseable que se les permitiera aplicar normas de origen más flexibles. Por último, deberían fortalecer su posición negociadora formando equipos de negociación conjuntos, en los que participen tanto quienes tengan un interés directo como todos los que, provenientes de los sectores productivos, puedan aportar su experiencia y sus conocimientos en ese campo.

(Traducido del inglés)

\section{Bibliografía}

Banco Mundial (1990): The Caribbean Common Market. Trade policies and regional integration in the 1990's, World Bank Report, $\mathrm{N}^{\circ}$ 8381-CRG, Washington, D.C., 21 de diciembre.

CEPAL (Comisión Económica para América Latina y el Caribe) (1995): Analisis preliminar de la relación entre el cambio arancelario y el comercio: impacto de la desgravación en el TLCAN en las exportaciones de los países del Istmo Centroamericano, Mexico D.F., Sede Subregional de la CEPAL en México.

De Vries, B. (1984): La política industrial en pequeñas economías en desarrollo: la determinación de una estrategia apropiada, Finanzas y desarrollo, vol. $21, \mathrm{~N}^{\circ} 2$, Washington, D.C., Fondo Monetario Internacional (FM)/Banco Mundial, junio.

Krugman, P y M. Obstfeld (1988): International Economics: Theory and Policy, Cambridge, Massachusetts, MIT Press.
Lewis, D. (1994): Assessment and review of the Central Ametican negotiations toward the NAFTA and their possible implications for CARICOM trade negotiation initiatives, Report on the North American Free Trade Agreement, Puerto España, G.S.R. Associates.

Markheim, D. (1994): A note on predicting the trade effects of economic integration and other preferencial trade agreements: An assessment, Journal of Common Market Studies, vol. 32, $\mathrm{N}^{\circ} 1$, Londres, Basil Blackwell Ltd., marzo.

Mc Intyre, A. (1994): An analysis of quantitative estimates of the trade and investment impacts of NAFTA, Report on the North American Free Trade Agreement, vol. Il, Puerto Espaffa, G.S.R. Associates.

Sera Puche, J. (1992): Conclusión de la negociación del TLC, El economista mexicano, Méxiço, D.F., Colegio Nacional de Economistas, octubre-diciembre. 